\title{
Haptic Negotiation and Role Exchange for Collaboration in Virtual Environments
}

\author{
S. Ozgur Oguz* \\ Ayse Kucukyilmaz \\ Tevfik Metin Sezgin \\ Cagatay Basdogan ${ }^{\S}$ \\ College of Engineering \\ Koç University
}

\begin{abstract}
We investigate how collaborative guidance can be realized in multimodal virtual environments for dynamic tasks involving motor control. Haptic guidance in our context can be defined as any form of force/tactile feedback that the computer generates to help a user execute a task in a faster, more accurate, and subjectively more pleasing fashion. In particular, we are interested in determining guidance mechanisms that best facilitate task performance and arouse a natural sense of collaboration. We suggest that a haptic guidance system can be further improved if it is supplemented with a role exchange mechanism, which allows the computer to adjust the forces it applies to the user in response to his/her actions. Recent work on collaboration and role exchange presented new perspectives on defining roles and interaction. However existing approaches mainly focus on relatively basic environments where the state of the system can be defined with a few parameters. We designed and implemented a complex and highly dynamic multimodal game for testing our interaction model. Since the state space of our application is complex, role exchange needs to be implemented carefully. We defined a novel negotiation process, which facilitates dynamic communication between the user and the computer, and realizes the exchange of roles using a three-state finite state machine. Our preliminary results indicate that even though the negotiation and role exchange mechanism we adopted does not improve performance in every evaluation criteria, it introduces a more personal and humanlike interaction model.
\end{abstract}

Index Terms: Human Factors; Evaluation/Methodology; Haptic I/O; Haptic User Interfaces; Haptic Guidance; Dynamic Systems and Control; Multimodal Systems; Virtual Environment Modeling; Human-computer interaction; Collaboration

\section{INTRODUCTION}

Although there has been substantial research in human-robot interaction in virtual environments as a research topic, little effort has been put into haptic guidance systems for collaborative tasks. By providing the user with appropriate feedback, haptics can improve task performance [8]. For many tasks, human-computer interaction requires collaboration, for which the user and the computer take on complementary and/or supportive roles. In this work, we adopt the collaboration definition given by Green et al.[5]: "working jointly with others or together especially in an intellectual endeavor". Such a collaboration scheme offers an exciting new way of interaction, in which computers can infer people's intentions and communicate with different people in different ways. It is worth noting that the utility of a collaborative system cannot be evaluated merely in terms

\footnotetext{
*e-mail:soguz@ku.edu.tr

†e-mail:akucukyilmaz@ku.edu.tr

*e-mail:mtsezgin@ku.edu.tr

§e-mail:cbasdogan@ku.edu.tr
}

IEEE Haptics Symposium 2010

25 - 26 March, Waltham, Massachusetts, USA

978-1-4244-6822-5/10/\$26.00 @2010 IEEE of performance and efficiency, but one should also take into account the quality of the interaction such as how much interaction can be realized and how comfortable and favorable the interaction is. This work is a preliminary study that investigates the benefits of guidance with collaborative role exchange mechanisms over simple guidance methods. Recent studies on collaborative dyadic interaction displayed the need to define certain roles for the partners $[9,13,2]$. However, defining the roles for a guidance scheme by examining human-human communication and replicating this interaction by replacing one of the dyads by the computer as a mean of providing guidance proves to be nontrivial especially as the task gets more complicated. In order to offer a comfortable experience in a dynamic complex environment, a sophisticated model is required. Collaboration is more than two partners working together. It requires defining a shared goal and in order to achieve this goal, two partners should create an agreement upon their courses of actions. Such an agreement is only achievable through negotiation. Our system employs a novel negotiation mechanism that realizes role exchange between a human and a computer partner using a three-state finite state machine. The primary advantage of the proposed scheme is that it creates a sense of togetherness while providing acceptable task performance. With this scheme, users can come up with different strategies and have the feeling of collaborating actively with another partner towards a common goal. Our initial findings suggest that using this scheme introduces a trade off between the accuracy in task performance and the effort of the user.

As a test bed application, we designed a multi-player haptic board game, where the user can share control with a computer partner. The user controls the position of a ball that can be moved on the board by tilting the board about two axes. The aim of the game is to hit randomly positioned targets in a specific order with the ball. The dynamic behavior of the game allows users to come up with different preferences. Some users felt comfortable in one of the axes and manipulated this axis more lightly and precisely than they did the other. Some regarded the order of the cylinders and moved very fast till they approached the target and then used the inertia of the ball to hit the target. These kinds of strategies requires the computer to provide guidance more actively. We designed a haptic guidance system in which the degree of computer's control can be varied independently in each axis during the performance of the task. In this system, the computer varies its level of participation in the task based on the actions of the user it collaborates with. We define certain roles for such a system where the user can either work collaboratively with the computer in equal terms or dominate the system. In other words, throughout the game, the computer and the user negotiate to take on control at varying levels. A role exchange occurs when the user's intention of gaining/releasing control is detected.

We designed an experiment to test the added benefit of our novel role exchange mechanism. In this experiment, we compare the performance of users in three conditions. In the first condition, the users play the game without guidance. In the second condition, guidance is provided, yet no negotiation takes place. Finally, the third condition implements our negotiation and role exchange 
mechanism. We quantify user performance and the utility of providing haptic guidance by measuring the task completion time, the deviation of the ball from the ideal path, integral of time and absolute magnitude of error (ITAE), and work done by the user. In addition, we evaluate the users' subjective self evaluation through a questionnaire.

In section 2, we briefly discuss related work on guidance in collaborative virtual environments and role determination in haptic communication. The architecture of the Haptic Board Game, certain guidance mechanisms that are provided to the users, and our negotiation model are presented in section 3, while the design of the experiment is discussed in section 4. Finally, the results of this study, which illustrate the potential benefits of negotiation and role exchange mechanisms in collaborative haptic guidance, and conclusions are presented in sections 5 and 6 , respectively.

\section{BACKGROUND}

The concept of haptic guidance is not new. In 1993, Rosenberg [10] came up with the concept of "virtual fixtures", which motivated many scientists to integrate haptics into human-computer interaction. A virtual fixture is defined as "abstract sensory information overlaid on top of reflected sensory feedback from a remote environment". Similar to a ruler guiding a pencil in line drawing, virtual fixtures are used to reduce mental processing and workload of certain sensory modalities as well as to improve precision and performance of the user beyond human capabilities. Virtual fixtures can help keep a task within a specific boundary using computer generated forces, and are often implemented using potential field and spring-damper systems. However, Forsyth and MacLean [4] report that these approaches can be problematic since the users' reactions towards the implemented guidance mechanism can cause oscillations within the system.

Several haptic guidance mechanisms are implemented to assist sensorimotor tasks, such as steering, calligraphy, and surgical training, and inclusion of haptics on top of existing modalities proved to be beneficial for training of such tasks. Recent work shows that haptics can be especially useful when combined with visual cues in teaching a sequence of forces [8]. Feygin at al. [3] conducted tests for spatio-temporal trajectory training and found out that the temporal aspects of the trajectory can be learned better with haptic guidance while visual training is more effective for learning the trajectory shape.

Although there have been some studies on haptic guidance and communication in shared virtual environments, only a few focused on defining roles for human-human and human-computer haptic collaboration. Sallnäs et al. [11] examined human-human collaboration for joint manipulation of a virtual object. They found out that haptic feedback significantly improves task performance and provides a better sense of presence in haptic collaboration. Basdogan et al. [1] proposed the haptic version of the "Turing Test" in their paper to better investigate the mechanisms of haptic interaction between two people in shared environments. They found out that haptic feedback provides a better sense of togetherness when compared to visual feedback.

Current systems involving computer guidance are generally implemented to let the human partner take on the leading role where the computer partner follows the human partner's actions [6, 7]. These prove to be beneficial in terms of task performance, yet are limited in providing a sense of collaboration since the computer is merely passive.

Reed and Peshkin [9] examine dyadic interaction to illustrate that partners specialize as accelerators and decelerators within a simple collaborative task. The specialization is said to be subconscious and occurs after several trials but improves performance. However applying the observed specialization scheme to the computer to collaborate with a human was not successful, probably due to a lack of careful examination of the negotiation process and how and why specialization occurs between dyads. Similarly, Stefanov et al. [13] propose execution and conductorship roles for haptic interaction. Specifically, the conductor decides what the system should do and expresses this intention via haptic signals, while the executor performs the actions as determined by the conductor. The conductor is assumed to express its intention by applying larger forces to the system. They suggest that by looking at the sign of the velocity and the interaction force, it is possible to determine which partner executes the task and propose a neat model for role exchange. They examine the phases of interaction that lead to different role distributions using a ternary logic, since each partner can take on one, both or none of the conductor or executor roles. This work presents important observations regarding communication for role exchange, yet employs no information on how this scheme can be used for human-computer interaction. Evrard et al. [2] similarly define leader and follower roles between which the partners continuously switch. In order to describe physical collaborative interaction, they use two distinct homotopy time functions that vary independently. Each partner can claim/give up leadership using these functions. For testing their model, they designed a symmetric dyadic task where a human interacts with a computer partner through an object. Despite the deficiencies in experimental design, they illustrate the potential use of homotopy functions in modeling different interaction behaviors. However unlike our approach, they have not implemented a user-centric and dynamic negotiation mechanism to handle the interaction between a human and a computer.

\section{HAPTIC BOARD GAME}

In this section, we describe the Haptic Board Game application as well as the guidance and the role exchange mechanisms we developed. For comparison, we tested the system under three conditions, namely no guidance, guidance without negotiation, and role exchange with negotiation. In the remainder of this chapter, we explain the general design approach we adopted and the application model used in implementing the conditions.

\subsection{Design Approach and Choice of Application}

We implemented an interactive game in a virtual environment in order to investigate how collaboration is achieved in dynamic environments and also to model and improve human-computer interaction. This game will be called the Haptic Board Game in the rest of this paper. Especially in dynamic, virtual, and shared worlds; it is not easy to program computers for providing generic assistance in interaction with the users. The Haptic Board Game involves controlling the position of a ball on a flat board to reach arbitrarily positioned targets with the help of a haptic device. The visual representation is reflected to the user as if the ball is moving by tilting the board about the $\mathrm{x}$ and $\mathrm{z}$ axes. The goal of the game is to hit 8 randomly placed cylinders with the ball in a specific order. At the beginning of a game, all the cylinders but the target are gray, and the target cylinder is highlighted with blue. When a user hits the target, its color turns red and the new target turns blue so that users can easily keep track of the current target, as well as the previous ones throughout the game (See Figure 1).

Our goal is to come up with a collaboration mechanism that can improve users' performance under this dynamic environment in terms of time, accuracy, and/or work done by the user; and also make them feel as if they are working with an intelligent entity. To achieve this, a force negotiation mechanism is developed, where each party can express his intentions and sense the other's. Since we are concerned with human-computer interaction, the computer should sense the user's intentions and act accordingly. Hence, we needed a model that provides more than simple automated com- 


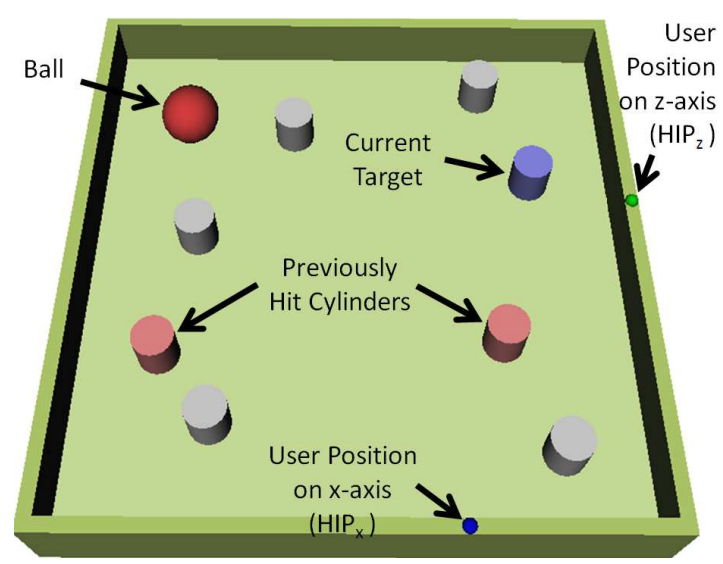

Figure 1: A screenshot of the Haptic Board Game. Red ball and randomly positioned eight cylindrical targets can be seen. The little half-spheres on the boundaries represent user controlled haptic device's position in $x-z$ plane. The haptic device's current position, in $x$ and $z$ axes referenced within the game frame, are indicated respectively by the blue and the green half-spheres.

puter guidance, and that can express intelligent reactions. Before conducting preliminary experiments, we implemented several models on how one should control the board and/or the ball. One of our initial designs included a system where the board was heavier and the ball's mass was negligible, letting the user feel the forces created by the inertia of the board. As another design, we modeled the board lighter and the ball heavier so that the user could feel the forces created by the ball's inertia more clearly. Yet, neither of these models, alone, met our expectations of creating a highly dynamic environment that can be realized by the user through both the visual and the haptic channels. Finally, we came up with a physical model (see Figure 2) that is more interaction oriented. More precisely, with this model users could feel not only the forces generated by the inertial movements of objects, but also those generated due to the haptic negotiation process with the controller. Moreover, the developed model provided us with a dynamic environment to test our hypotheses. Different parameter sets providing various guidance and collaboration mechanisms, were also investigated to optimize the system. The details of this model will be explained later on this section.

While experimenting on the choice of the system model, three conditions were tested on each design:

Both Axes Guidance (BG): Both the user and the computer have control on both axes, and each affects the system equally. Both axes guidance is implemented with a classical PD (Proportional-Derivative) control algorithm. The controller adjusts the orientation of the board such that the ball automatically moves towards the target. The user feels the forces applied to the ball by the controller and the resistive spring-like forces due to his/her actions. The user can affect the behavior of the ball, while the computer guidance is given regardless of the user's interventions.

Role Exchange (RE): The computer negotiates with the user, based on the user's force profile, to decide on how they should share control. The magnitude of computer control can be either equal to that of the user's or smaller. When partners share control equally, this condition becomes identical to both axes guidance. On the other hand, when the computer control switches to a rather loose level, the computer's forces are reduced, hence the user becomes dominant on controlling the ball while the computer becomes the recessive partner. In between these states, computer's control is blended from equal control to looser control or vice versa.

No Guidance (NG): The user feels spring-like resistive forces due to the rotation of the board, but no haptic guidance is given to control the ball position on the board.

\subsection{Physical Model and Conditions}

Considering our hypotheses and observations, we devised a novel negotiation model for role exchange and compared it to one of the classical control methods, namely PD control, implemented as both axes guidance $(\mathrm{BG})$ condition in our experiments. Additionally in the role exchange (RE) condition, the degree of provided guidance is adjusted dynamically via a role exchange policy. In our role exchange policy, we model the force negotiation between the user and the computer using a simple mass-spring-damper system. In this system, the ball is controlled by three virtual control points as shown in Figure 2: Haptic interface point (HIP), controller interface point (CIP), and negotiated interface point (NIP) which are all regarded as massless particles. HIP, CIP and the ball are interconnected at NIP, which is the only element that interacts directly with the ball.

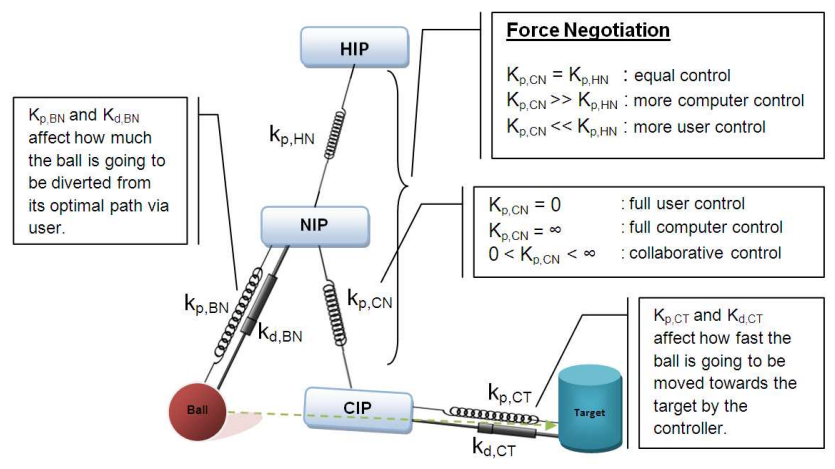

Figure 2: The physical model for role exchange and both axes guidance conditions. $K_{p}$ and $K_{d}$ values in the figure represent the spring and damper coefficients.

\subsubsection{Both Axes Guidance}

For both axes guidance, the system is basically controlled by haptic and controller interface points. The flow diagram of the physical model of the game is shown in Figure 3. Users control the haptic interface point by a PHANToM Omni (SensAble Technologies Inc.) haptic device, whereas controller interface point is controlled by the PD control algorithm. When guidance is provided, at any given time, the controller computes an optimal force $\left(F_{c}\right.$ in Figure 3) as if to control the ball. However, rather than applying this force directly to the ball, it is applied to the system through controller interface point; so that controller interface point moves towards the target and pulls the ball to itself. Hence, in the lack of user interference, the controller can easily control the ball, and play the game smoothly. The user participated in the task by controlling haptic interface point in order to move the ball. The user applies a force to the system through haptic interface point. Based on the new positions of haptic and controller interface points, the position of negotiated interface point can be calculated to put the system into equilibrium. The forces that act on negotiated interface point, due to controller's and the user's interventions, are $F_{C I P}$ and $F_{H I P}$, respectively (see Figure 3 ). Negotiated interface point can be thought as the position of the ball agreed by both parties. Also, the ball also applies a force, $F_{b a l l}$, on negotiated interface point, due to the spring-damper system modeled between negotiated interface point and itself. Therefore, the new equilibrium position of negotiated interface point, for the next time step $(t+\Delta t)$, is calculated according 
to the net forces acting on it. The force that would act on the ball, $F_{b a l l}$, is determined by the position of the ball and the new position of negotiated interface point. As illustrated in Figure 3, the board is oriented in order to provide the needed force, $F_{b a l l}$, to be applied on the ball. Then, the ball's new state can be calculated based on the orientation of the board, by Euler integration. Finally, the force, $\left(-F_{H I P}\right.$ in Figure 3$)$, created by the spring system between negotiated and haptic interface points is fed back to the user. Hence, by this physical interaction flow, the dynamic nature of the Haptic Board Game is reflected to the user through both the visual and the haptic channels.

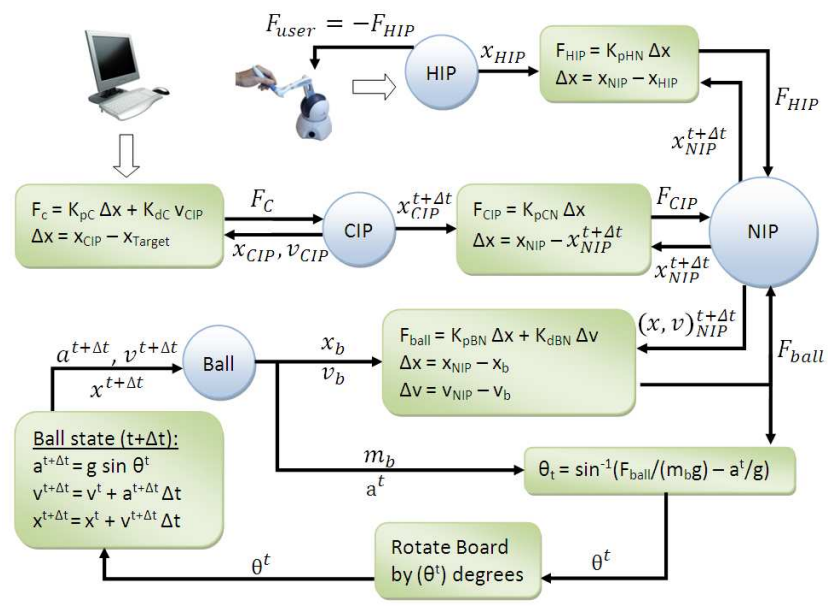

Figure 3: The flow of interactions within the Haptic Board Game's physical model. $x, v$, and $a$ represent position, velocity, and acceleration of the ball, respectively. $g$ is the gravitational acceleration, whereas $\theta$ is the orientation angle of the board.

\subsubsection{Role Exchange}

The interaction points and the physical model of interaction for role exchange is identical to those of both axes guidance condition. However, role exchange takes a step further by dynamically changing the role of the controller, i.e. the degree of control it provides. The system is designed to allow haptic negotiation between partners by sensing the user's intentions. For this purpose, the user's average forces and the standard deviation of the forces on each axis are calculated under no guidance condition at the beginning of the experiments (see Section 4 for details). Then, lower and upper force threshold values are calculated for each axis using the average force and standard deviation of the user playing the game.

It is assumed that role exchange occurs whenever the magnitude of the force that the user applies is above the upper threshold or below the lower threshold values for over a predetermined amount of time. This amount is fixed as 500 milliseconds in our implementation. In order to realize a smooth transition during role exchange, we defined a finite state machine with three states as shown in Figure 4. Initially the system is in the user dominant state, in which the user is mainly in control of the game, while the controller gently assists him. If the force applied by the user stays below the calculated lower threshold value for $90 \%$ of the last 500 milliseconds, then the controller assumes that the user requires more assistance. Thus, role exchange occurs in favor of the computer and the system enters role blending state in which the computer gradually takes control until its level of control reaches that of the user's. The system stays in the role blending state for a period of 1000 milliseconds. After this period, the system enters the equal control state, where the system acts identically as in both axes guidance condition. Clearly, another state transition may occur from the equal control state to the role

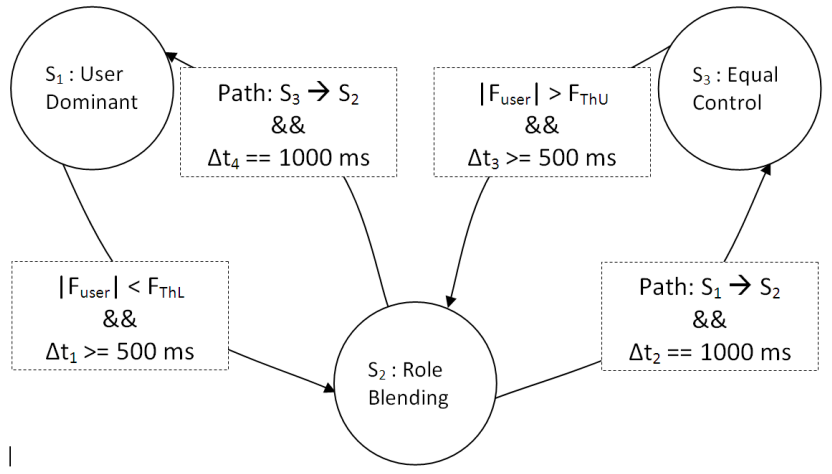

Figure 4: The state diagram defining the role exchange policy. $F_{\text {user }}$ is the force that the user applies. $F_{T h L}$ and $F_{T h U}$ are the lower and upper threshold values, respectively, for determining the state transitions. $S_{1}$ : User dominant state indicates user is the dominant actor, and $S_{3}$ : Equal control state indicates both computer and user have equal degree of control on the system. Finally, $S_{2}$ : Role blending state indicates a role exchange blending phase is taking place where controller's role is shifted between user dominant and equal control states.

blending state, if the controller realizes that the user wants to take over control. Then, the same procedure applies but in the opposite direction where the computer releases control and the user becomes the dominant actor of the system.

As stated earlier, in both axes guidance condition, the computer shares control with the user throughout the game to help the user complete the task by providing guidance in both axes of the board, based on the position and direction of the ball. However, in our preliminary studies, one of the observations was that the force profiles of users on each axis did not show similar patterns. For instance, a user could have preferred to be attentive in one axis and aligned the ball on that axis first, then she/he switched her/his attention and tried to control the ball on the other axis. Hence, we concluded that the users did not pay attention to both axes equally at the same time. This may be due to the random positioning of the target cylinders, i.e. some consecutive targets were positioned diagonally, whereas some were in parallel to each other on one axis. Another possible reason can be that the users might not feel comfortable controlling the ball diagonally and prefer a sequential control on axes. Hence, we extended our role exchange method by allowing state transitions to occur on each axis separately. In other words, computer can give full guidance on one axis whereas it just remains recessive on the other and let the user remain the dominant actor on that axis. An example of this state transitions can be seen in Figure 5. For example, at the fifth second, a transition occurs from user dominant state to role blending state for the $\mathrm{x}$-axis, i.e the controller starts to get more control on the x-axis. Around one-half of a second later, another state transition occurs from user dominant state to role blending state again, but on z-axis. Spending one second on role blending state, another transition from role blending to the equal control state takes place, first for $\mathrm{x}$-axis then for $\mathrm{z}$-axis. At around sixth second of playing, controller becomes as effective as the user for controlling the ball, hence the condition becomes identical to both axes guidance. By allowing role exchange on each axis separately, this condition becomes more adaptive to differences between users' playing styles. Finally, the user feels a spring-like force that is generated due to the positions of haptic and negotiated interface points, like in the both axes guidance condition. Notice that users can feel the controller's applied forces, as well as the transitions that it makes, through negotiated interface point. For example, assume the controller is in equal control state, so that the negotiated interface point lies just in the middle of controller and haptic interface points if they do not happen to coincide on the same position 

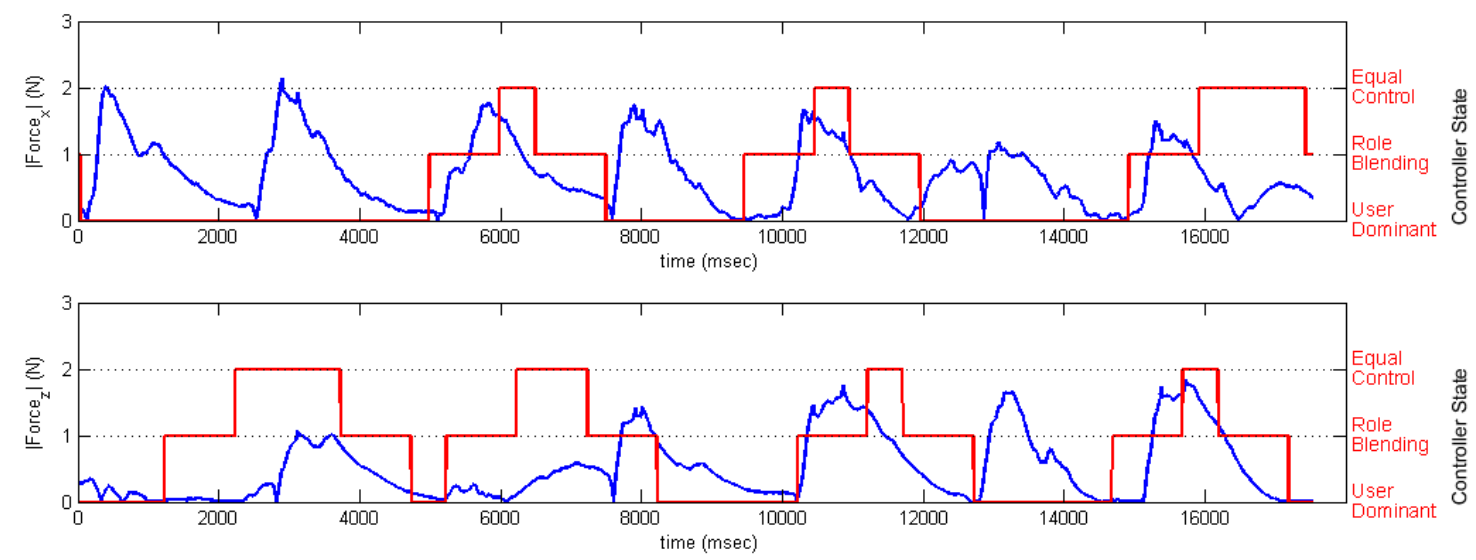

Figure 5: A cross-section of a user's force values in each axis throughout a single game with role exchange. The state of the controller, which shows the current role of the controller in the related axis, is represented as a square waveform. The role is determined by the state variable indicated on right hand side $y$-axis. The upper and lower plots represent the information in $x$ and $z$ axes respectively.

(the inertial forces of the ball is neglected for the sake of simplifying the example). Hence, the user feels a conflicting force. If the user and the controller have discordant preferences, this conflicting situation continues, which eventually enforces the controller to enter the role blending, and then the user dominant states. Since the controller loosens its control, the negotiated interface point starts to be pulled by haptic interface point stronger. As negotiated interface point moves closer to haptic interface point, the force that is fed to the user decreases, alleviating the conflicting situation. Moreover, due to the blending phase between user dominant and equal control states, users may feel a smooth transition, so that they do not get distracted by the role transitions of the controller.

\subsubsection{No Guidance}

Finally, as the base case, we implemented the Haptic Board Game with no guidance. In this condition, controller interface point basically coincides with negotiated interface point and is never disconnected from it. Therefore, only haptic interface point affects negotiated interface point, which in turn pulls the ball towards itself. In other words, the model in Figure 3 does not produce $F_{C I P}$, but the remaining forces continue acting on the system. As a result, the user feels $-F_{H I P}$ due to the spring system between haptic and negotiated interface points.

\section{EXPERIMENT}

\subsection{Objectives and Approach}

We sought an indication of the effectiveness and acceptability of a negotiated haptic interaction method, as modeled by the role exchange condition, relative to the classical PD controller based guidance and to no guidance at all, for performing dynamic tasks. The main hypotheses that we aimed to test were:

H1 Role exchange has measurable benefits over other conditions.

H2 Users will subjectively prefer role exchange over other conditions.

\subsection{Experiment}

10 subjects ( 5 female, and 5 male) participated in our study ${ }^{1}$. In order to eliminate learning effects on successive trials, the order

${ }^{1}$ After an initial analysis, we found that one subject did not report any sensation of computer control in the questionnaire, therefore the remaining questions, which were about the nature of computer control, were rendered inapplicable. Hence, we excluded his responses from further analysis, and analyzed the remaining 9 users for all conditions. of experimental conditions was mixed, with at least three days between two successive experiments.

Since none of our users were familiar with a haptic device, we introduced the haptic device to each user verbally and through the use of certain training applications irrelevant to the board game. Each user utilized these applications for about 15 minutes until they felt comfortable with the haptic device. An experiment took about half an hour, and in each experiment the users played with either no guidance, both axes guidance, or guidance with role exchange. We paid attention to provide the same physical setting for all experiments, such as the positioning of the haptic device, the computer, and the users' seats. Subjects were instructed to grasp the stylus in the most effective and comfortable way possible. During the experiments, the full system state (i.e. positions of HIP, CIP, NIP, and ball; all the individual forces of each spring/spring-damper system, etc.) was recorded at $1 \mathrm{kHz}$.

In the no guidance and both axes guidance conditions, each user played the haptic board game 15 times for a single experiment. As explained earlier in Section 3, a single game consists of hitting the ball to eight randomly placed cylinders in a specific order, by controlling the ball. When a single game finishes, all the cylinders turn gray again, and the game restarts without interrupting the system's simulation. To avoid possible fatigue, users took a break after the $5^{\text {th }}$ and the $10^{\text {th }}$ games. For the role exchange condition, the users played an additional game at the beginning of each block of 5 games for the purpose of determining the thresholds, so a total of 18 games were played by each user. During these extra games, users played with no guidance. In order to create the user's force profile, the average and the standard deviation of the user's forces were calculated during these first games, so that the lower and upper threshold values could be determined for the next 5 games.

\subsection{Metrics}

\subsubsection{Subjective Evaluation Metrics}

After each experiment, the users were given a questionnaire. Users did not know about the different conditions we were testing, nor did they know whether they took these experiments with different conditions or not.

For the questionnaire design, we adopted the technique that Slater et al. used previously in shared visual environments [12]. A total of 18 questions were answered by the subjects. Eight of the questions were about personal information, one was reserved for users' feedback and the remaining nine were about variables directly related to our investigation. Some of the questions were paraphrased, and asked again, but scattered randomly in the ques- 
tionnaire. For evaluation, the averages of these questions, that fall into the same category, were calculated. Questions were asked in five categories:

1. Performance: Each user was asked to assess his performance by rating himself on a 5 -point Likert scale.

2. Human-likeness: We asked the subjects whether the control felt through the device, if any, was humanlike or not. Two questions using a 7-point Likert scale were included within the questionnaire.

3. Collaboration: We asked the subjects whether they had a sense of collaborating with the computer or not. Two questions with different wordings were asked within the questionnaire. Two more questions were asked to determine whether the control made it harder for the subjects to complete the task or not. Answers to these 4 questions were evaluated using a 7-point Likert scale.

4. Degree of User Control: We asked the subjects about their experience during the experiment, specifically the perceived degree of their control on the task. There was a single question, which used a 7-point Likert scale for the answer.

5. Degree of Computer Control: We asked the subjects about the perceived degree of computer's control on the task. There was a single question, which used a 7-point Likert scale for the answer.

\subsubsection{Objective Performance Metrics}

User performance can be quantified in terms of task completion time, total path length during the game, deviation of the ball from the ideal path and integral of time and absolute magnitude of error (ITAE).

For the board game, we defined the ideal path between two targets to be the straight line segment connecting the centers of the targets. Hence, between two targets, the deviation is defined to be the area of the region formed by the ideal path between those targets and the actual path of the ball. Total deviation in a single game is calculated by summing the deviations between consecutive targets throughout the course of the game.

ITAE criterion is defined as:

$$
I T A E=\sum_{i=1}^{7}\left(\int_{t=T_{i}}^{T_{i+1}} t|e(t)| d t\right) .
$$

Note that we calculate ITAE for consecutive target pairs and sum these to get the ITAE of a game. Here, time $T_{i}$ is taken as the moment when the ball reaches $i^{t h}$ target. Error $e(t)$ is the length of the shortest line segment connecting the ideal path and the ball's actual position at time $t$ during the game. The ITAE criterion has the advantage of penalizing the errors that are made later. In other words, we choose to punish the users more severely if they deviate from the path when the ball gets close to hitting the target.

We also examined work done by the user due to the spring located between NIP and HIP. This spring acts as the bridge between the system and the haptic device and any force exerted by it is sent indirectly to the user. Hence we assume that this force is the force felt by the user. Let $T$ be the completion time of the game, $k$ be the stiffness constant of the spring, and $x(t)$ be the extension of the spring at time $t$. Then the work done by the spring is basically:

$$
W=\int_{t=0}^{T} \frac{1}{2} k x(t)^{2}
$$

\section{RESULts}

\subsection{Subjective Evaluation Results}

For each of the three conditions, the questionnaire was designed to measure the self-perception of users' performance, the humanlikeness and the collaborative aspects of the system, as well as the degree to which the users felt they or the computer were in control.

For the level of perceived collaboration, the subjective evaluation results implied a higher sense of collaboration for the role exchange and both axes guidance conditions $(p<0.01)$ compared to the no guidance condition. There was no significant difference between the level of perceived collaboration in both axes guidance and role exchange conditions (see Figure 6).

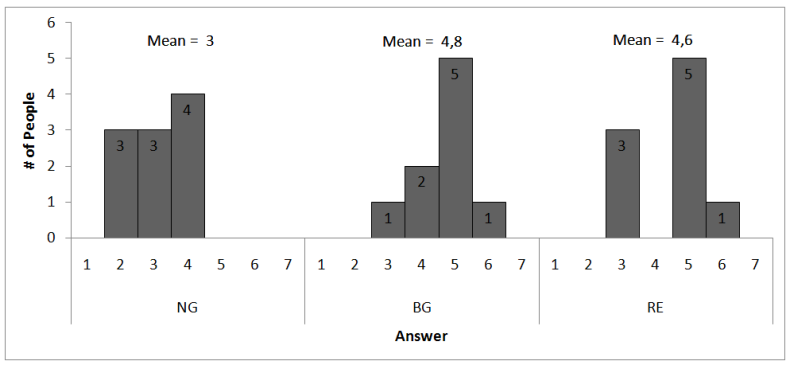

Figure 6: Responses to questions regarding how much the subjects felt collaboration for each condition.

Regarding the subjective evaluation of user performances, subjects believed that they performed better on both axes guidance and role exchange compared to the no guidance condition. The differences were statistically significant for both axes guidance and role exchange when compared to the no guidance condition with the p-values of 0.005 and 0.02 , respectively. Again, there is no significant difference between the both axes guidance and role exchange cases (see Figure 7). Subjects claimed that they had similar level of control throughout the game in all three conditions. On the other hand, they also felt no difference between the level of computer control on different conditions. However, regarding the averages of the answers to the control questions, we observed that the subjects' feeling of being in control and their perception of computer's involvement get closer to each other in role exchange condition, as illustrated in Figure 8. Even though the subjects perceived reduced control over the game, they had a stronger sense of computer participation. This may also be a sign of subjects' increased perception of collaboration in the role exchange condition.

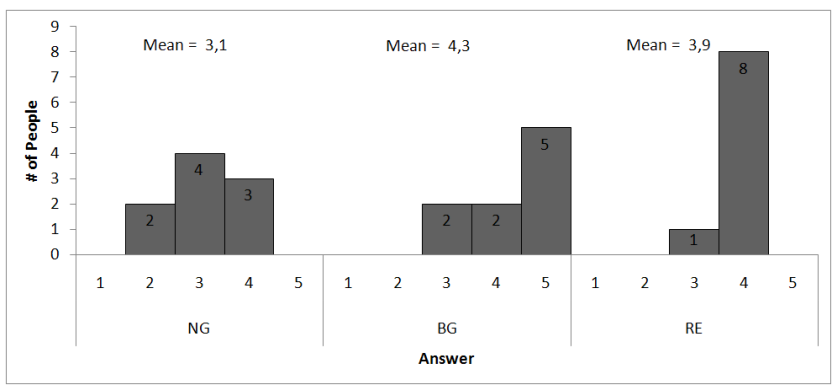

Figure 7: Responses to questions regarding subjects' self evaluation of how well they performed in each condition.

Finally, regarding the humanlikeness question, subjects did not think there was a significant difference between both axes guidance and role exchange conditions. On the other hand, subjects felt that role exchange condition's negotiation strategy was more humanlike $(p$-value $=0.02)$ compared to no guidance condition (see Figure 9). 
Our negotiation model allows role exchange and provides the controller with the ability to take over/release the control of the game.

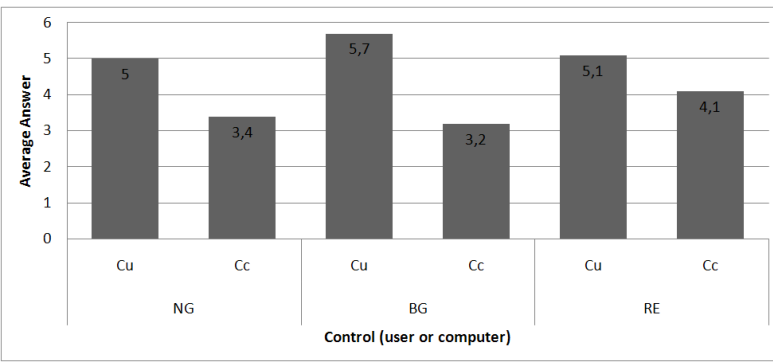

Figure 8: Average responses to questions regarding how much the subjects felt in control, and how much they felt the computer was in control for each condition. $\mathrm{Cu}$ and $\mathrm{Cc}$ represent control of user, and control of computer, respectively.

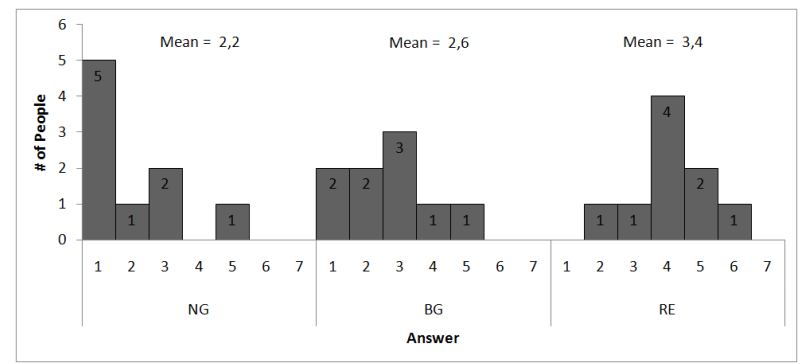

Figure 9: Responses to questions regarding how much the subjects felt humanlike response for each condition.

\subsection{Quantitative Measurements}

We computed the average computation times, total path lengths, deviations from the ideal path, and ITAEs of each condition. Upon closer inspection, we observed that performance is the worst when no guidance is given, and the best when guidance without any negotiation, while guidance with the role exchange mechanism falls in between the two. We applied paired t-tests, with $p$-value set to 0.05 , to test the difference between the conditions. According to paired t-test results all three conditions display significant difference from each other. As seen in Figure 10, for all these parameters, the paired differences of conditions follow a similar trend. Clearly, the largest difference is between the no guidance and both axes guidance conditions. We also observe that the role exchange and both axes guidance conditions are the closest conditions regarding the paired differences.

Figure 11 illustrates the average energy on the spring, which is a measure of the work done by the user. Even though in the no guidance and role exchange conditions, the completion time and path errors were higher compared to the both axes guidance condition, the users spent less energy in these conditions. The paired t-test results on the average work done by the user did not indicate significant difference between the no guidance and role exchange conditions, whereas both are statistically different from the both axes guidance condition. As the results above show, both axes guidance has higher energy requirements, while no guidance has inferior completion time and spatial error properties. The role exchange mechanism allows us to trade off accuracy for energy without causing user dissatisfaction.

We also examined the role exchange trends of users. As seen in Figures 12 and 13, the results show that the average number of transitions as well as the average time the controller stays at a given state varies from user to user. This is a sign of the existence of user
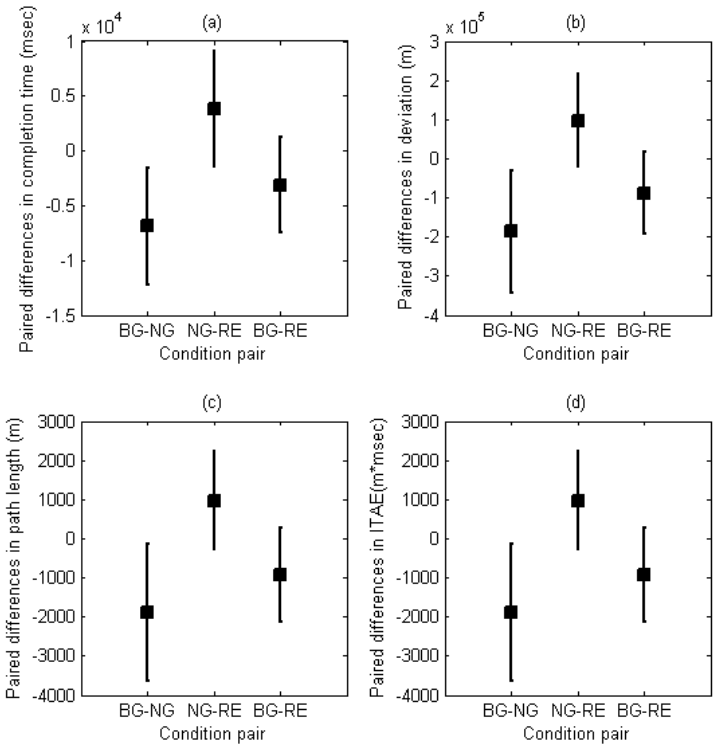

Figure 10: Means and standard deviations of paired differences of (a) computation times, (b) path deviations, (c) path lengths, and (d) ITAEs per condition (NG: no guidance, BG: guidance without negotiation, $\mathrm{RE}$ : guidance with negotiation and role exchange)
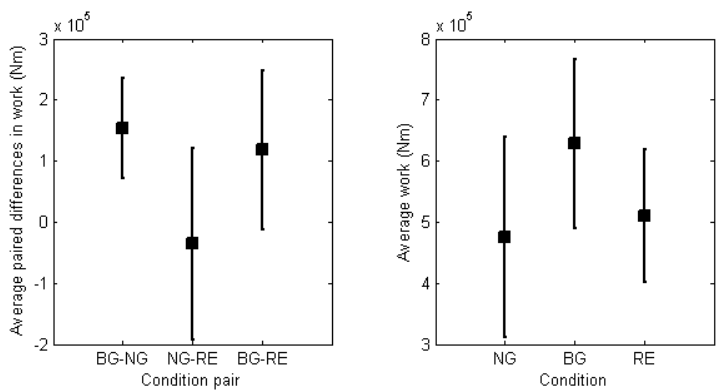

Figure 11: Means and standard deviations of energy on the spring between NIP and HIP per condition and paired differences of energy (NG: no guidance, BG: guidance without negotiation, RE: guidance with negotiation and role exchange)

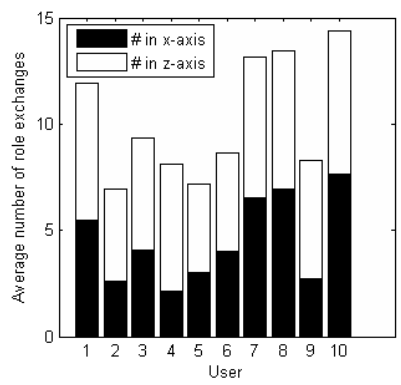

Figure 12: Average number of role exchanges of each user over 15 games. Each user ends up with a different number of role exchanges, indicating that they adopt certain strategies during the course of the game.

preferences during game play. Even though subjective evaluations suggest that the development of these preferences is subconscious, this is a strong indication that our role exchange mechanism provides a more personal experience compared to classical guidance mechanisms. 


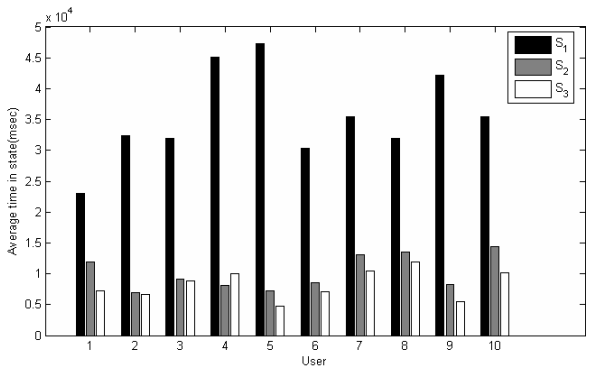

Figure 13: Average time spent by users in each controller state. S1, $\mathrm{S} 2$, and S3 represent user dominant, role blending, and equal control states respectively, as depicted in Figure 4

\section{CONCLUSION}

In this paper, we developed a model for haptic negotiation and role exchange between a human user and a computer in a collaborative task. Our model works in a highly dynamic setting and aims to realize collaboration naturally and without disturbing the user. The nature of our task forced us to build a sophisticated dynamic negotiation mechanism between the user and the computer. Furthermore, we defined the role exchange mechanism using a finite state machine that allowed us to realize fluid interaction. As the results imply, with our role exchange mechanism, the users are presented with an option to choose and optimize between accuracy and energy.

\section{FUtURE WORK}

In the current experimental setting, we did not inform the users about the mechanisms that were tested. Hence the users were not made aware of the existence of the states of the role exchange mechanism. As future work, we intend to extend this experiment to let users play the game with a priori knowledge about the existence of the different conditions, so that we can better evaluate our collaboration scheme.

The current system implements a specific negotiation and role exchange mechanism. We'd like to use the Haptic Board Game application as a test bed for developing and testing alternative negotiation and role exchange methods. For example, we'd like to explore the potential use of sophisticated machine learning based methods for detecting user intent for initiating negotiation. Likewise, we'd like to study how the use of accompanying multimodal displays would effect the dynamics of role exchange and negotiation.

We would also like to carry out further experiments tailored to measure aspects of the interaction that we haven't studied yet. For example, teasing out the precise cause of the perceived humanlikeness is a nontrivial task that we haven't addressed here.

\section{ACKNOWLEDGEMENTS}

\section{REFERENCES}

[1] C. Basdogan, C.-H. Ho, M. A. Srinivasan, and M. Slater. An experimental study on the role of touch in shared virtual environments. $A C M$ Trans. Comput.-Hum. Interact., 7(4):443-460, 2000.

[2] P. Evrard and A. Kheddar. Homotopy switching model for dyad haptic interaction in physical collaborative tasks. In WHC '09: Proceedings of the World Haptics 2009 - Third Joint EuroHaptics conference and Symposium on Haptic Interfaces for Virtual Environment and Teleoperator Systems, pages 45-50, Washington, DC, USA, 2009. IEEE Computer Society.

[3] D. Feygin, M. Keehner, and F. Tendick. Haptic guidance: Experimental evaluation of a haptic training method for a perceptual motor skill. In HAPTICS '02: Proceedings of the 10th Symposium on Haptic Interfaces for Virtual Environment and Teleoperator Systems, page 40, Washington, DC, USA, 2002. IEEE Computer Society.

[4] B. A. C. Forsyth and K. E. MacLean. Predictive haptic guidance: Intelligent user assistance for the control of dynamic tasks. IEEE Transactions on Visualization and Computer Graphics, 12(1):103113, 2006.

[5] S. Green, M. Billinghurst, X. Q. Chen, and G. Chase. Human robot collaboration: An augmented reality approach a literature review and analysis. In MESA 07, 3rd International Conference on Mechatronics and Embedded Systems and Applications, Nevada, USA, 2007.

[6] O. Khatib. Mobile manipulation: The robotic assistant. Robotics and Autonomous Systems, 26(2-3):175-183, 1999.

[7] K. Kosuge, H. Yoshida, and T. Fukuda. Dynamic control for robothuman collaboration. In Robot and Human Communication, 1993. Proceedings., 2nd IEEE International Workshop on, pages 398-401, Nov 1993.

[8] D. Morris, H. Tan, F. Barbagli, T. Chang, and K. Salisbury. Haptic feedback enhances force skill learning. In WHC '07: Proceedings of the Second Joint EuroHaptics Conference and Symposium on Haptic Interfaces for Virtual Environment and Teleoperator Systems, pages 21-26, Washington, DC, USA, 2007. IEEE Computer Society.

[9] K. B. Reed and M. A. Peshkin. Physical collaboration of humanhuman and human-robot teams. IEEE Trans. Haptics, 1(2):108-120, 2008.

[10] L. Rosenberg. Virtual fixtures: Perceptual tools for telerobotic manipulation. In Virtual Reality Annual International Symposium, 1993. 1993 IEEE, pages 76-82, Sep 1993.

[11] E.-L. Sallnäs, K. Rassmus-Gröhn, and C. Sjöström. Supporting presence in collaborative environments by haptic force feedback. ACM Trans. Comput.-Hum. Interact., 7(4):461-476, 2000.

[12] M. Slater, A. Sadagic, M. Usoh, and R. Schroeder. Small-group behavior in a virtual and real environment: A comparative study. Presence: Teleoper. Virtual Environ., 9(1):37-51, 2000.

[13] N. Stefanov, A. Peer, and M. Buss. Role determination in humanhuman interaction. In WHC '09: Proceedings of the World Haptics 2009 - Third Joint EuroHaptics conference and Symposium on Haptic Interfaces for Virtual Environment and Teleoperator Systems, pages 51-56, Washington, DC, USA, 2009. IEEE Computer Society. 